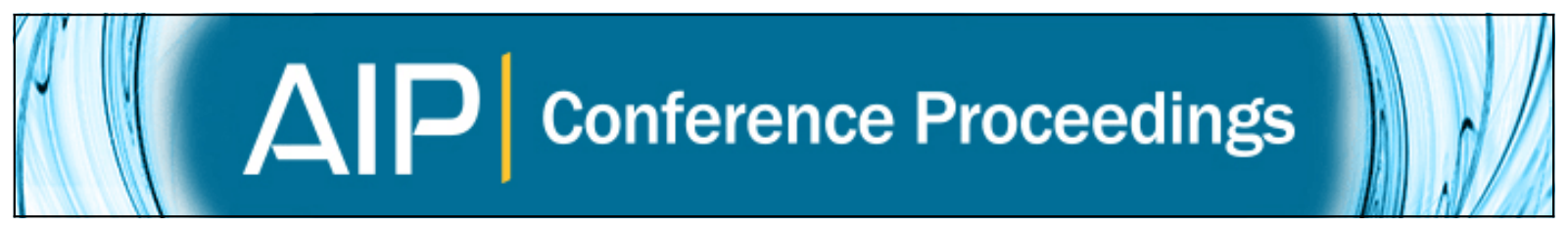

\title{
Field localization in a modified Randall-Sundrum brane model
}

Triyanta, D. Singleton, P. Jones, and G. Munoz

Citation: AIP Conference Proceedings 1617, 96 (2014); doi: 10.1063/1.4897113

View online: http://dx.doi.org/10.1063/1.4897113

View Table of Contents: http://scitation.aip.org/content/aip/proceeding/aipcp/1617?ver=pdfcov

Published by the AIP Publishing

\section{Articles you may be interested in}

Localization of a system of vector and scalar fields in a modified Randall-Sundrum model

AIP Conf. Proc. 1677, 040003 (2015); 10.1063/1.4930647

Randall-Sundrum cosmological model with nonminimal derivative coupling of scalar field AIP Conf. Proc. 1656, 050006 (2015); 10.1063/1.4917125

Flavor and Higgs Physics in Randall-Sundrum Models

AIP Conf. Proc. 1116, 418 (2009); 10.1063/1.3131588

How a Randall-Sundrum Brane-World Effective Potential Influences Inflation Physics AIP Conf. Proc. 880, 1170 (2007); 10.1063/1.2437564

Dynamical Stability of the AdS Soliton in Randall-Sundrum Model

AIP Conf. Proc. 646, 203 (2002); 10.1063/1.1524574 


\title{
Field Localization in a Modified Randall-Sundrum Brane Model
}

\author{
Triyanta $^{1}$, D. Singleton ${ }^{2}$, P. Jones ${ }^{3}$, and G. Munoz ${ }^{2, *}$ \\ ${ }^{I}$ Division of Theoretical High Energy Physics and Instrumentation, Physics Department, Faculty of Mathematics \\ and Natural Sciences, Institut Teknologi Bandung and \\ Indonesian Center for Theoretical and Mathematical Physics \\ Jalan Ganesha 10 Bandung 40132, Indonesia \\ ${ }_{2}^{2}$ Physics Department, California State University Fresno, 2345 East San Ramon Avenue M/S 37, Fresno, California \\ 93740-8031 \\ ${ }^{3}$ Physics Department, Cal Poly, San Luis Obispo, CA 93407 \\ *Email:triyanta@fi.itb.ac.id
}

\begin{abstract}
We consider a modified Randall-Sundrum metric model in a five-dimensional spacetime. The metric is conformally flat, has an exponential warp factor, and is physically distinct from the original Randall-Sundrum metric model. Massless scalar fields are localized on the brane for decreasing warp factor while massive scalar fields are localized on the brane for either decreasing or increasing warp factors. There is an upper bound mass for the decreasing warp factor and there are two possible different masses for the increasing warp factor. For a constant value of the fifth component of the vector matter field we obtain a possible localization for massless vector fields, but not for massive vector fields. Both massless and massive spinor fields unfortunately are not localized on the brane.
\end{abstract}

Keywords: Extra dimension, field localization, five-dimensional brane model, Randall-Rundrum.

PACS: $11.10 . \mathrm{Kk}, 04.50 .-\mathrm{h}$

\section{INTRODUCTION}

Theories with higher dimensional spacetimes have been among the most interesting research topics in theoretical physics since the beginning of the modern physics era. Kaluza-Klein theory $[1,2]$ introduced an extra dimension to unify electromagnetic and gravitational forces. In braneworld models [3-10] extra dimensions are used to address hierarchy problems. Such a model hypothesizes that ordinary matter is localized on a brane, a four-dimensional spacetime, which is imbedded in a bulk, higher-dimensional spacetime. The Randall-Sundrum model $[5,10]$ is among the most popular brane world models with one extra dimension (the $y$-coordinate). The first type of Randall-Sundrum model uses two branes, the Planck brane at $y=0$ and the $\mathrm{TeV}$ brane at $y=2 \pi R$ where $R$ is the bulk radius. The second type of model uses only one brane located at $y=0$. The five-dimensional spacetime in the Randall-Sundrum model is defined through the following metric

$$
(d s)^{2}=e^{-2 k|y|} \eta_{\mu v} d x^{\mu} d x^{v}+(d y)^{2} .
$$

In the above $k$ is a constant and $\eta_{\mu \nu}$ is the Minkowski metric. By defining the new extra coordinate $z$ through $(d y)^{2}=\exp (-2 k|y|)(d z)^{2}$ [9] the above metric can be written as

$$
(d s)^{2}=\frac{1}{(k|z|+1)^{2}} \eta_{A B} d x^{A} d x^{B}
$$

where $A, B=0,1,2,3,5$ with $z$ as the fifth coordinate. Unlike the metric (1) this metric has the same warp factor for all coordinates, but it does not have an exponential form. We can define a new metric which is conformally flat like (2) but with an exponential warp factor like (1) [11]

$$
(d s)^{2}=e^{-2 k|r|} \eta_{A B} d X^{A} d X^{B} .
$$

In the above $r$ is the extra coordinate, $X^{5}=r$. It turns out that this $r$ metric differs from the Randall-Sundrum metric (1) in the extra coordinate part: (3) has an exponential warp factor while (1) has a constant, unity, warp factor. Thus we can say that (3) is a modified Randall-Sundrum metric. In fact we can obtain (3) from (1) through $(d y)^{2}=\exp (-2 k|r|)(d r)^{2}$ and $\exp (-k|r|) d X^{\mu}=\exp (-k|y|) d x^{\mu}$ [11]. Spacetimes defined by the metrics (1) and (2) are similar, their proper distances along the extra dimension is infinite. The spacetime defined by (3) on the other hand differs from (1) and (2). Depending on the values of $k$

3rd International Conference on Theoretical and Applied Physics 2013 (ICTAP 2013)

AIP Conf. Proc. 1617, 96-99 (2014); doi: 10.1063/1.4897113

(C) 2014 AIP Publishing LLC 978-0-7354-1254-5/ $\$ 30.00$ 
spacetime (3) has either infinite or finite proper distance along extra dimension. Also, the Ricci scalars of the metric (1) and (2) are the same but they differ from (3). The fine tuning of the metrics (1) and (2), i.e. setting the cosmological constant $\lambda=-k^{2}$, leads to the vanishing of the energy-momentum tensors in the bulk for metrics (1) and (2) but not for metric (3) [11,12].

\section{LOCALIZATION OF MATTER FIELDS}

We consider in this section the spacetime metric (3). For convenience we write $X^{\mu}$ as $x^{\mu}$ and $X^{5}$ as $r$. The action for a complex scalar field $\Phi$ is given by

$$
S_{0}=\int d^{5} x \sqrt{g} g^{M N} \partial_{M} \Phi * \partial_{N} \Phi .
$$

Decomposing $\Phi\left(x^{\mu}, r\right)=\varphi\left(x^{\mu}\right) \chi_{0}(r)$ leads to

$$
\begin{aligned}
S_{0}= & \left(\int_{0}^{\infty} d r \sqrt{g} g^{\mu v} \chi_{0}^{*} \chi_{0}\right) \int d^{4} x \partial_{\mu} \varphi^{*} \partial_{\nu} \varphi+ \\
& \left(\int_{0}^{\infty} d r \sqrt{g} g^{r r} \partial_{r} \chi_{0}^{*} \partial_{r} \chi_{0}\right) \int d^{4} x \varphi^{*} \varphi .
\end{aligned}
$$

The condition for localization is that the two integrals over $r$ in the above equation should be finite. Normalizing the field $\varphi$, the first integral equals unity while the second integral equals $m^{2}$, where $m$ is the mass of the scalar field on the brane. Both integrals depend on $\chi_{0}(r)$. This function is the solution of the equation of motion of the field in the bulk and has the form [11]

$$
\chi_{0}= \begin{cases}b_{0} e^{3 k r}+c_{0} & m=0 \\
e^{\frac{3}{2} k r}\left(\begin{array}{l}
b_{0} e^{+\frac{1}{2} r \sqrt{9 k^{2}-4 m^{2}}}+ \\
c_{0} e^{-\frac{1}{2} r \sqrt{9 k^{2}-4 m^{2}}}
\end{array}\right) & m \neq 0\end{cases}
$$

Inserting the above solution into the $r$-integrals in (5) one can conclude that: 1) for the massless mode the field $\varphi$ is localized on the brane only for decreasing warp factor $(k>0)$ with $\left.b_{0}=0,2\right)$ for the massive mode the field $\varphi$ is localized for both decreasing and increasing warp factors when we set $c_{0}=0$. This is better localization as compared to the original RandallSundrum model where massive scalar field cannot be confined. Note that for the massive mode for both decreasing and increasing warp factors the mass of the scalar field fulfils

$$
m^{2}=k^{2} \frac{11 \pm \sqrt{13}}{8}
$$

and, in addition, for decreasing warp factor there is an upper bound mass of $\sqrt{ } 5 k / 2$. Thus for decreasing warp factor the scalar field has one possible mass $m=k \sqrt{(11-\sqrt{13}) / 8}$ and for increasing warp factor the field has two possible masses $m_{-}=|k| \sqrt{(11-\sqrt{13}) / 8}$ and $m_{+}=|k| \sqrt{(11+\sqrt{13}) / 8}$. By setting the decreasing warp factor in the modified Randall-Sundrum model consistent with a $126 \mathrm{GeV}$ localized scalar Higgs boson [13] one can predict a second scalar boson at $177 \mathrm{GeV}$ [12]. Alternatively, setting the increasing warp factor in the modified Randall-Sundrum model such that $m_{+}=126 \mathrm{GeV}$ one can predict a scalar boson at $90 \mathrm{GeV}$.

Now we analyze the localization properties of massless vector fields $A^{M}$ with the action

$$
S_{1}=\int d^{5} x \frac{-1}{4} \sqrt{g} g^{M N} g^{R S} F_{M R} F_{N S} .
$$

Decomposing $A_{\mu}\left(x^{M}\right)=a_{\mu}\left(x^{\nu}\right) c(r) \quad$ and $\quad$ setting $A_{r}=$ constant the field equation corresponding to the above action is equivalent to the Maxwell's equation (equation for massless vector fields) on the brane

$$
\partial^{\mu} f_{\mu v}=0, \quad f_{\mu v}=\partial_{\mu} a_{v}-\partial_{v} a_{\mu} .
$$

There are two possible cases for $\left.c(r): 1) c(r)=c_{1}, 2\right)$ $c(r)=d_{1} \exp (k r)$ where $c_{1}$ and $d_{1}$ are constants of integration. The constant solution corresponds to any gauge choices while the non-constant solution corresponds to the Lorentz gauge. For the first case the $r$-integral in the action is finite for decreasing warp factor and infinite for increasing warp factor. This means that for the constant solution and decreasing warp factor the vector fields are localized. This is a better result as compared to the Randall-Sundrum model. For the second case the $r$-integral in the action is also finite but it leads to non-zero masses. This is inconsistent with the massless Maxwell field equation above. Thus localization for the non-constant solution $c(r)$ is not possible.

Next, considering a massive vector fields $A^{M}$ in the five-dimensional spacetime, we should add into the integrand of the action $S_{1}$ in (8) a mass term of the form

$$
\frac{1}{2} m^{2} \sqrt{g} g^{M N} A_{M} A_{N}
$$


The Euler-Lagrange equation corresponding to the action from (8) gives the field equation

$$
\partial_{M}\left(\sqrt{g} g^{M N} g^{R S} F_{N S}\right)+m^{2} \sqrt{g} g^{R N} A_{N}=0
$$

which is equivalent to

$$
\begin{aligned}
0= & \left(\partial^{\alpha} a_{\alpha}\right) \partial_{5} c(r)-m^{2} e^{-2 k r} A_{5}, \\
0= & \eta^{\nu \beta} c(r)\left[\partial^{\alpha} f_{\alpha \beta}+m^{2} e^{-2 k r} a_{\beta}\right] \\
& +\eta^{\nu \alpha} a_{\alpha}\left[k \partial_{5} c(r)-\partial_{5}{ }^{2} c(r)\right] .
\end{aligned}
$$

Recalling that $x^{\mu}$ and $r$ are independent of one another, equation (i) gives $A_{5}=0$ and either $c(r)$ is a constant or $c(r)$ is not a constant but should fulfill the Lorentz gauge $\partial^{\alpha} a_{\alpha}=0$. For a constant $c(r)$, equation (ii) is equivalent to Proca's equation, instead of Maxwell's equation (9) on the brane. For a non-constant $c(r)$, equation (ii) is also equivalent to Proca's equation with $c(r)=d_{1} \exp (k r)$. Thus both massless and massive cases relate to the same solution of $c(r)$. Note that the mass of $a^{\mu}$ on the brane $r=0$ is equal to $m$ and it increases or decreases by the warp factor. To analyze the localization properties for massive vector fields we write the action $S_{1}$ (i.e. eq. (8) after adding the mass term (10)) by separating the $r$-integrals from other integrals using the field decomposition $A^{\mu}\left(x^{M}\right)=$ $a^{\mu}\left(x^{V}\right) c(r)$. The final expression is

$$
\begin{aligned}
S_{1}= & -\frac{1}{4} \int_{0}^{\infty} d r e^{-k r} c(r)^{2} \int d^{4} x \eta^{\mu v} \eta^{\alpha \beta} f_{\mu \alpha} f_{v \beta} \\
& +\frac{1}{2} \int_{0}^{\infty} d r e^{-k r}\left(\partial_{r} c(r)\right)^{2} \int d^{4} x \eta^{\mu v} a_{\mu} a_{v} \\
& +\frac{m^{2}}{2} \int_{0}^{\infty} d r e^{-3 k r} c(r)^{2} \int d^{4} x \eta^{\mu v} a_{\mu} a_{v} .
\end{aligned}
$$

For a constant $c(r)$ and $k>0$ the second term of the above equation vanishes and the other $r$-integrals are finite. Next, the first $r$-integral is equal to $c_{1}{ }^{2} / k$ and thus gives $c_{1}^{2}=k$. The third $r$-integral is equal to $c_{1}^{2} / 3 k$ and this defines the mass of the vector field $a^{\mu}$ : $M=m / 3$. This value of mass is inconsistent with the mass according to Proca's equation (which is equal to $m$ on the brane). Thus we conclude that for a constant $c(r)$ and $k>0$ the field cannot be localized. For constant $c(r)$ and $k<0$ the first and third $r$-integrals are infinite so for this case the field also cannot be localized. Because the first two $r$-integral are infinite for nonconstant $c(r)$ and $k>0$ and the third $r$-integral is infinite for $\mathrm{k}<0$ we conclude that the vector field cannot be localized for non-constant $c(r)$. So for all cases the massive vector fields cannot be confined on the brane.

Finally we come to spinor fields. The corresponding action is

$$
S_{1 / 2}=\int d^{5} x \sqrt{g} \bar{\Psi} i \Gamma^{M} D_{M} \Psi .
$$

In the above, $\Gamma^{M}$ are the curved space Dirac matrices and for the corresponding metric, the covariant derivative $D_{M}$ are [11]

$$
D_{\mu}=\partial_{\mu}+\frac{i k}{2} \gamma_{\mu} \gamma_{5}, \quad D_{r}=\partial_{r} .
$$

By decomposing the 5D spinor field as

$$
\Psi\left(x^{\mu}, r\right)=\left(\begin{array}{l}
\psi_{R}\left(x^{\mu}\right) p_{R}(r) \\
\psi_{L}\left(x^{\mu}\right) p_{L}(r)
\end{array}\right)
$$

the field equation derived from the action $S_{1 / 2}$ gives the following solutions for $p_{R, L}[11]$ :

$$
\begin{aligned}
& p_{R}(r)=c_{1 / 2} \exp (2 k r-m r), \\
& p_{L}(r)=d_{1 / 2} \exp (2 k r+m r) .
\end{aligned}
$$

The coefficients $c_{1 / 2}$ and $d_{1 / 2}$ are constants of integration. With these solutions the $r$-integrals in the action have the forms

$$
\begin{gathered}
\left(c_{1 / 2}\right)^{2} \int_{0}^{\infty} \mathrm{e}^{-2 m r} d r, \\
\left(d_{1 / 2}\right)^{2} \int_{0}^{\infty} \mathrm{e}^{2 m r} d r, \\
c_{1 / 2} d_{1 / 2} \int_{0}^{\infty} d r .
\end{gathered}
$$

For massive modes $(m \neq 0)$, the first expression is finite while the second and third expressions are infinite, except when we set $d_{1 / 2}=0$. But the third expression is part of the mass term on the brane so it cannot be zero for the massive mode. For massless fields $(m=0)$ all the above expressions are infinite except when both $c_{1 / 2}$ and $d_{1 / 2}$ are set to vanish, i.e. if there is no spinor field. Thus because all the three expressions above cannot be finite all together we conclude that spinor fields are not localized on the brane. Better localization cannot be obtained for a massive spinor field $\Psi$ in the five-dimensional space because the $r$ integral in this additional term cannot cancel the third $r$-integral above. In [14] it was found that spinor field localization can be achieved by introducing an 
unrealistic imaginary mass term in the fivedimensional spacetime. One might try to localize the spinor field by taking the spinor field $\Psi$ to interact with a scalar field $\Phi$ through a Yukawa coupling $g \bar{\Psi} \Psi \Phi$. We leave this investigation for future work.

\section{CONCLUSIONS}

The metric (3) that we considered is derivable from the Randall-Sundrum metric (1) via a relationship of the form $(d y)^{2}=\exp (-2 k|r|)(d r)^{2}$ and $\exp (-k|r|) d X^{\mu}$ $=\exp (-k|y|) d x^{\mu}$ between the fifth coordinates $y$ and $r$ of both metrics. However there are some distinct features between both metrics. Both metrics have different Ricci scalars, Einstein tensors and energy-momentum tensors. The proper distance into the bulk for the metric (1) is infinite while for metric (3) it depends on the warp factor. Decreasing warp factor in metric (3) gives finite proper distance into the bulk and increasing warp factor gives infinite proper distance.

Scalar and vector matter fields can be localized on the brane $r=0$ for the metric (3) for the following conditions: Massless scalar-fields are localized on the brane for a decreasing warp factor but not for an increasing warp factor, the same localization property for the metric (1). However, while massive scalar fields are not localized for the Randall-Sundrum metric (1) they can be localized on the brane for the metric (3) for either case, decreasing or increasing warp factors. In addition, there is a mass upper bound on the scalar fields for a decreasing warp factor and there are two possible masses for an increasing warp factor.

Massless vector fields can be localized for a decreasing warp factor and a constant $c(r)$. This is a better result when compared to the original RandallSundrum model. For non-constant $c(r)$, massless vector fields are not localized, either for decreasing or increasing warp factors. Also massive vector fields cannot be localized on the brane for any kind of warp factor.

Finally, we found that massless and massive spinor fields cannot be localized on the brane. Thus there is no improved localization for the spinor case, as compared to the metric (1). To seek possible localization for spinor fields we must then look at nongravitational interactions. Introducing a Yukawa coupling between a scalar field confined to the brane and the spinors fields is one type of interactions that is worth considered. We will look at this possibility in future work.

\section{ACKNOWLEDGEMENTS}

Triyanta was supported by the Riset dan Inovasi ITB 2013.

\section{REFERENCES}

1. T. Kaluza, Sitzungsber. Preuss. Akad. Wiss. Berlin (Math. Phys.) K1,966 (1921);

2. O. Klein, Z. Phys. 37, 895 (1926).

3. N. Arkani-Hamed, S. Dimopoulos, and G. Dvali, Phys.Lett. B 429, 263 (1998).

4. Antoniadis, N. Arkani-Hamed, S. Dimopoulos, and G.Dvali, Phys. Lett. B 436, 257 (1998).

5. L. Randall and R. Sundrum, Phys. Rev. Lett. 83, 3370 (1999).

6. M. Gogberashvili, Int. J. Mod. Phys. D 11, 1635 (2002)

7. M. Gogberashvili, Int. J. Mod. Phys. D 11, 1639 (2002).

8. M. Gogberashvili, Mod. Phys. Lett. A 14, 2025 (1999).

9. M. Gogberashvili, Europhys. Lett. 49, 396 (2000).

10. L. Randall and R. Sundrum, Phys. Rev. Lett. 83, 4690 (1999).

11. P. Jones, G. Munoz, D. Singleton, and Triyanta, Phys. Rev. D 88, 025048 (2013)

12. P. Jones, G. Munoz, D. Singleton, and Triyanta, DPF2013-84, September, arXiv:1309.4790 (2013)

13. ATLAS collaboration, Phys. Lett. B 716 1-29 (2012), arXiv:hep-th/0407276v1.

14. I. Oda, Phys. Lett. B 508, 96(2001). 